\title{
Generating ultrashort coherent soft x-ray radiation in storage rings using angular-modulated electron beams
}

\author{
D. Xiang \\ SLAC National Accelerator Laboratory, Menlo Park, CA, 94025, USA \\ W. Wan \\ Lawrence Berkeley National Laboratory, Berkeley, CA, 94720, USA
}

(Dated: February 2, 2010)

\begin{abstract}
A technique is proposed to generate ultrashort coherent soft x-ray radiation in storage rings using angular-modulated electron beams. In the scheme a laser operating in the TEM01 mode is first used to modulate the angular distribution of the electron beam in an undulator. After passing through a special beam line with non-zero transfer matrix element $R_{54}$, the angular modulation is converted to density modulation which contains considerable higher harmonic contents of the laser. It is found that the harmonic number can be one or two orders of magnitude higher than the standard coherent harmonic generation method which relies on beam energy modulation. The technique has the potential of generating femtosecond coherent soft x-ray radiation directly from an infrared seed laser and may open new research opportunities for ultrafast sciences in storage rings.
\end{abstract}

Work supported in part by US Department of Energy contract DE-AC02-76SF00515. 
Over the years electron storage rings (SR) have played a great role in x-ray sciences. In $\mathrm{SR}$, the circulating relativistic electron beams generate $\mathrm{x}$-rays in the bending magnets and in the insertion devices which have wide applications in physics, biology, chemistry, etc. The electron bunch length in SR is typically on the order of a few tens of ps which limits the duration of the x-ray pulses and the x-ray radiation is incoherent in time. There is growing interest in generating ultrashort x-ray pulses with good temporal coherence in SR: the ultrashort pulse can break the ps time barrier and enable the study of ultrafast dynamic processes such as chemical bond forming and breaking, phase transitions in solids, etc; and the coherent pulse is highly desirable for high-resolution spectroscopy, high-resolution imaging, etc.

Unlike the linac-based light sources where electron beam with high peak current and small energy spread can be used to drive a seeded single pass free electron laser, the beams in SR typically have a relatively low current and large energy spread which is unable to initiate the exponential gain of the radiation power in the undulator. Therefore, most of the methods to generate ultrashort coherent radiation in SR use the coherent harmonic generation (CHG) technique. In the standard CHG scheme [1-3], a seed laser with wavelength $\lambda$ is first used to generate energy modulation in the beam in a modulator. After passing through a dispersive element, the energy modulation is converted to density modulation. Finally the densitymodulated beam is used to generate coherent radiation at the wavelength $\lambda / n$ in the radiator, where $n$ is an integer and is called the harmonic number.

The CHG technique has been applied in the SR to generate coherent radiation in the ultraviolet region $[1,4,5]$. Typically generating the $n$th harmonic of the seed laser requires the energy modulation amplitude to be approximately $n$ times larger than the beam energy spread. Due to the inherent large energy spread of the beam in SR, the harmonic number from the standard CHG scheme is limited to $3 \sim 5$, about one order of magnitude lower than what required to reach soft $\mathrm{x}$-ray region.

In this paper, we propose a scheme to extend the harmonic number in CHG by an order of magnitude using angular-modulated electron beams in SR. The proposed scheme is based on the fact that although the balance between quantum excitation and radiation damping in SR results in a relatively large energy spread, it also provides a beam with extremely low vertical emittance. Our proposed method makes full use of this natural gift and it is shown that one can achieve a much higher harmonic number by modulating the vertical divergence 


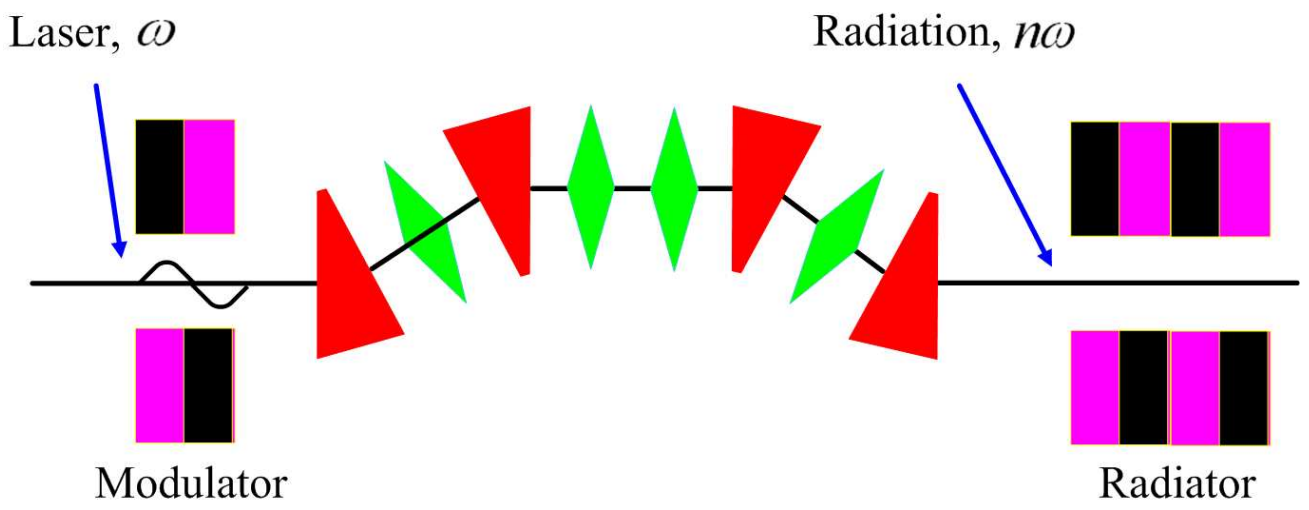

FIG. 1: Scheme for generation of ultrashort coherent soft x-ray radiation using angular-modulated electron beams in SR.

of the beam as compared to the standard case where the beam energy is modulated. With representative realistic beam parameters, we show that one can generate coherent $16 \mathrm{~nm}$ radiation in SR directly from an $800 \mathrm{~nm}$ seed laser $(n=50)$ with existing technologies. The proposed method has the potential of extending the wavelength of the coherent radiation down to soft x-ray region and may open new research opportunities for ultrafast sciences in SR.

The proposed scheme for generation of ultrashort soft x-ray radiation in SR is shown in Fig. 1. An intense infrared laser $(\lambda=800 \mathrm{~nm})$ operating at TEM01 mode with the duration of about $100 \mathrm{fs}$ is first used to interact with the electron beam in the undulator (modulator) resonant to the laser frequency to generate angular modulation in the beam. A beam line consists of four dipole magnets (red triangles in Fig. 1) and four quadrupole magnets (green diamonds in Fig. 1) is used to convert the angular modulation into density modulation. The beam is then sent through another undulator (radiator) tuned to the harmonic frequency of the laser to generate coherent radiation at a shorter wavelength. The radiation pulses are temporally and transversely coherent, with a duration comparable to that of the seed laser. Such a beam line can be put in the long straight sections of the SR and can act as an useful add-on to enhance the capabilities of SR in ultrafast sciences.

To make full use of the low vertical emittance in SR, the beam should wiggle in the vertical plane and interact with the TEM01 mode laser so that the beam's vertical divergence is modulated in the modulator. The electric field for the TEM01 mode laser with constant 
power may be written as [6],

$$
\begin{aligned}
E_{y}= & \frac{E_{0}}{1+\left(z / z_{0}\right)^{2}} \frac{2 \sqrt{2} y}{w_{0}} \sin (k(z-c t)+\phi) \\
& \times \exp \left[-\frac{x^{2}+y^{2}}{w_{0}^{2}\left(1+\left(z / z_{0}\right)^{2}\right)}\right] .
\end{aligned}
$$

where $E_{0}$ is the laser field amplitude, $\lambda=2 \pi / k$ is the laser wavelength, $w_{0}$ is the waist size, $z_{0}=\pi w_{0}^{2} / \lambda$ is the Rayleigh length and $\phi=2 \pi s / \lambda-2 \tan ^{-1}\left(z / z_{0}\right)+\pi z\left(x^{2}+y^{2}\right) /\left(\lambda\left(z^{2}+z_{0}^{2}\right)\right)$ is the phase seen by the electron at longitudinal position $s$ with respect to the reference particle. When the laser waist size is much larger than the electron beam size, one can neglect the last term in Eq. (1) and the term with $x$ and $y$ dependence in the phase expression. After interaction with the TEM01 laser, the electron's energy and divergence changes as [6],

$$
\begin{aligned}
& \Delta \gamma(x, y, s) / \gamma=A k y \cos (k s), \\
& \Delta y^{\prime}(x, y, s)=A \sin (k s) .
\end{aligned}
$$

where $A=\left(2 K / \gamma^{2}\right) \sqrt{P / P_{0}}[J J] f, P$ is the laser power, $P_{0}=I_{A} m c^{2} / e, I_{A}$ is the Alfvèn current, $[J J]=J_{0}(\xi)-J_{1}(\xi)$ with $\xi=K^{2} /\left(4+2 K^{2}\right), f$ is a function depending on the undulator length and the Rayleigh length.

To convert the vertical angular modulation into longitudinal density modulation, a beam line which couples the beam's motion in vertical and longitudinal planes is needed. The coupling is typically initiated by bending the beam in vertical direction. Various beam line may be used to convert the angular modulation into density modulation. In our example, we use a representative beam line which consists of a chicane and four quadrupoles, as shown in Fig. 1. Hereafter we will only consider the motion in $y-z$ plane and neglect the uncoupled motion in horizontal plane. We also assume the beam is much longer than the laser wavelength, so that we can neglect the current variation within several laser wavelength. Consider a beam with vertical rms divergence value $\sigma_{y}^{\prime}$, the initial distribution of the beam can be written as $f_{0}\left(y_{p}\right)=N_{0} / \sqrt{2 \pi} \exp \left(-y_{p}^{2} / 2\right)$, where $y_{p}=y^{\prime} / \sigma_{y}^{\prime}$ is the dimensionless divergence for some particle, $N_{0}$ is the number of electrons per unit of length. After interaction with the TEM01 laser, the particles's vertical dimensionless divergence $y_{p, 1}$ is related to its initial value by $y_{p, 1}=y_{p}+B \sin (k s)$, where $B=\Delta y^{\prime} / \sigma_{y}^{\prime}$ is the normalized angular modulation amplitude. The beam's distribution after interaction with the laser becomes,

$$
f_{1}\left(y_{p}, s\right)=\frac{N_{0}}{\sqrt{2 \pi}} \exp \left[-\frac{\left(y_{p}-B \sin (k s)\right)^{2}}{2}\right] .
$$


The electron's state in phase space is generally characterized by $\overrightarrow{g_{0}}=$ $\left(x, x^{\prime}, y, y^{\prime}, z, \Delta E / E\right)^{T}$. After passing through a linear Hamiltonian system, the electron's state $\overrightarrow{g_{0}}$ becomes $\overrightarrow{g_{1}}=R \overrightarrow{g_{0}}$, where $R$ is a $6 \times 6$ symplectic matrix (see, for example [7]) which describes the beam dynamics associated with the system. Consider the beam line shown in Fig. 1, analysis shows that by properly choosing the strength for the quadrupoles, one can make all the elements $R_{5 i}$ of the transfer matrix vanishing except for $i=4$ and $i=5$ $\left(R_{55}=1\right)$. In this case, due to the symplectic condition and the mirror symmetry, one finds that the elements $R_{4 j}$ are all zero except for $j=4\left(R_{44}=-1, R_{33}=-1\right.$ as well). Then, to the first order, after passing through the beam line, particle's longitudinal position $s_{2}$ is related to the position at the entrance to the chicane $s_{1}$ and the dimensionless divergence at the entrance to the chicane $y_{p, 1}$ by $s_{2}=s_{1}+R_{54} \sigma_{y}^{\prime} y_{p, 1}$ and the divergence also changes to $y_{p, 2}=-y_{p, 1}$. Then the beam's distribution evolves to,

$$
f_{2}\left(y_{p}, s\right)=\frac{N_{0}}{\sqrt{2 \pi}} \exp \left(-\frac{\left(y_{p}+B \sin \left(k s-r y_{p}\right)\right)^{2}}{2}\right) .
$$

where $r=k \sigma_{y}^{\prime} R_{54}$. Integration of Eq. (4) over $y_{p}$ gives the beam current distribution which can be expanded into Fourier series,

$$
N(s)=N_{0}\left[1+2 \sum_{n=1}^{\infty} b_{n} \sin \left(n k s+\phi_{n}\right)\right] .
$$

where bunching factor for the $n$th harmonic is found to be [2],

$$
b_{n}=e^{-\frac{1}{2} n^{2} r^{2}}\left|J_{n}(n B r)\right|
$$

The bunching factor has a similar form as that in standard CHG scheme. In both schemes, generation of the $n$th harmonic requires the normalized modulation amplitude to be approximately $n$ times larger than the divergence or energy spread of the beam.

In a $\mathrm{SR}$, since the energy spread of the beam $\sigma_{E}$ is typically on the order of a few $\mathrm{MeV}$, it is difficult to extend the harmonic number to $n>10$ using the standard CHG technique. First, the required laser power scales as the square of the modulation amplitude, so the required laser power grows quickly as the harmonic number increases. Second, the beam energy spread will increase to approximately $B \sigma_{E} / \sqrt{2}$ after the energy modulation. The increased energy spread may significantly perturb the nominal operation of the SR if it exceeds some threshold. Due to these limitations, the harmonic number achieved in experiments using the standard CHG method is limited to about $n=3$. The shortest wavelength of the coherent 
radiation generated in a SR using the standard CHG methods is $132 \mathrm{~nm}$ from a $396 \mathrm{~nm}$ seed laser [5].

Although the balance between quantum excitation and radiation damping results in a relatively large energy spread, it nontheless provides a beam with very low vertical emittance as a natural gift. Our proposed method makes full use of this feature and thus has the potential to extend the harmonic number to a much higher value. From Eq. (2) it follows that the rms energy spread growth of the beam after the interaction with the TEM01 laser is $\Delta \gamma=\sqrt{2} B \gamma \epsilon_{y} / \lambda$ which greatly depends on the vertical emittance. It can be anticipated that if the beam has an extremely low vertical emittance, one can achieve a very large $B$ while still limiting the energy spread growth within permissible level.

To show the application of our proposed scheme in generating ultrafast coherent soft $\mathrm{X}$-rays in SR, we use the beam parameters for the proposed PEP-X light source at SLAC [8] as a representative example. The beam parameters are listed in Table. 1. To have a small vertical emittance, we assume that the coupling parameter is $1 \%$ for the SR and the geometric vertical emittance is $2 \mathrm{pm}$ [8]. With this extremely low vertical emittance, the energy spread growth is only about $3.5 \mathrm{MeV}$ (smaller than the intrinsic energy spread) for $B=70$ when modulated by an $800 \mathrm{~nm}$ laser, which is in sharp contrast to the standard CHG scheme where for the same normalized modulation amplitude the energy spread increases to about $250 \mathrm{MeV}$.

In our example we assume the modulator has 6 periods with a period length of $25 \mathrm{~cm}$ and the seed laser has a wavelength of $800 \mathrm{~nm}$. The dipole magnet in the chicane has a length of $0.1 \mathrm{~m}$ and a bending angle of about 0.12 degrees. The distance between the first and second dipole (and hence that between the third and forth dipoles) is $0.6 \mathrm{~m}$ and that between the second and third dipoles is $1.65 \mathrm{~m}$. The quadrupole strengths are properly adjusted to achieve vanishing $R_{53}$ and $R_{56}$. The corresponding $R_{54}$ for the chicane is about $-6.47 \mathrm{~mm}$. The radiator has 25 periods with a period length of $8 \mathrm{~cm}$ and is tuned to the 50 th harmonic of the seed laser (wavelength at $16 \mathrm{~nm}$ ).

The laser-beam interaction is simulated following Eq. (2), which is justified when the laser waist size is much larger than the electron beam size. Assuming the laser waist is $0.5 \mathrm{~mm}$ and the laser power of about $6.3 \mathrm{TW}$ is needed in order to generate an angular modulation amplitude 70 times larger than the beam's intrinsic vertical divergence. In reality the required TEM01 mode can be excited with standard mode-matching technique 
and the required TW laser power can be obtained with standard chirped pulse amplification techniques. Such kind of high power laser is widely used in inverse Compton scattering experiment [9] and laser wake field acceleration experiment [10]. The beam is then tracked through the beam line using the code ELEGANT [11] with second order transport effects taken into account.

The distributions of the beam in $y^{\prime}-z$ space along the beam line are shown in Fig. 2 . After interaction with the laser, the beam's angular distribution is modulated by the TEM01 laser, as can be seen in Fig. 2a. After passing through the chicane, the angular modulation is converted to density modulation as shown in Fig. 2b. Note the divergence changes sign after passing through the chicane, which is due to the fact that $R_{44}=-1$ for the beam line. The energy spread of the beam increases from $5 \mathrm{MeV}$ to about $6.4 \mathrm{MeV}$, which should not significantly affect the beam dynamics in the SR.

The bunching factor for the harmonic number $n$ is calculated as $\left\langle e^{i n k s}\right\rangle$, where $n k$ is the wave number of the harmonic radiation and the brackets denote averaging over the

TABLE I: Main parameters for the coherent soft x-ray radiation generation using angularmodulated electron beams

$\begin{array}{lc}\text { Electron beam energy } & 4.5 \mathrm{GeV} \\ \text { Peak current } & 300 \mathrm{~A} \\ \text { Geometric horizontal emittance } & 0.2 \mathrm{~nm} \mathrm{rad} \\ \text { Geometric vertical emittance } & 2 \mathrm{pm} \mathrm{rad} \\ \text { Relative energy spread } & 1.14 \mathrm{e}-3 \\ \text { Intrinsic vertical divergence } \sigma_{y}^{\prime} & 0.283 \mu \mathrm{rad} \\ R_{54} \text { of the beam line } & -6.47 \mathrm{~mm} \\ N_{p} \times \lambda_{u} \text { for the modulator } & 6 \times 25 \mathrm{~cm} \\ \text { Laser wavelength in the modulator } & 800 \mathrm{~nm} \\ \text { Laser waist size in the modulator } & 0.5 \mathrm{~mm} \\ \text { Peak laser power in the modulator } & 6.3 \mathrm{TW} \\ N_{p} \times \lambda_{u} \text { for the radiator } & 25 \times 8 \mathrm{~cm} \\ \text { Radiation wavelength in the radiator } & 16 \mathrm{~nm} \\ \text { Peak radiation power in the radiator } & 3 \mathrm{MW}\end{array}$



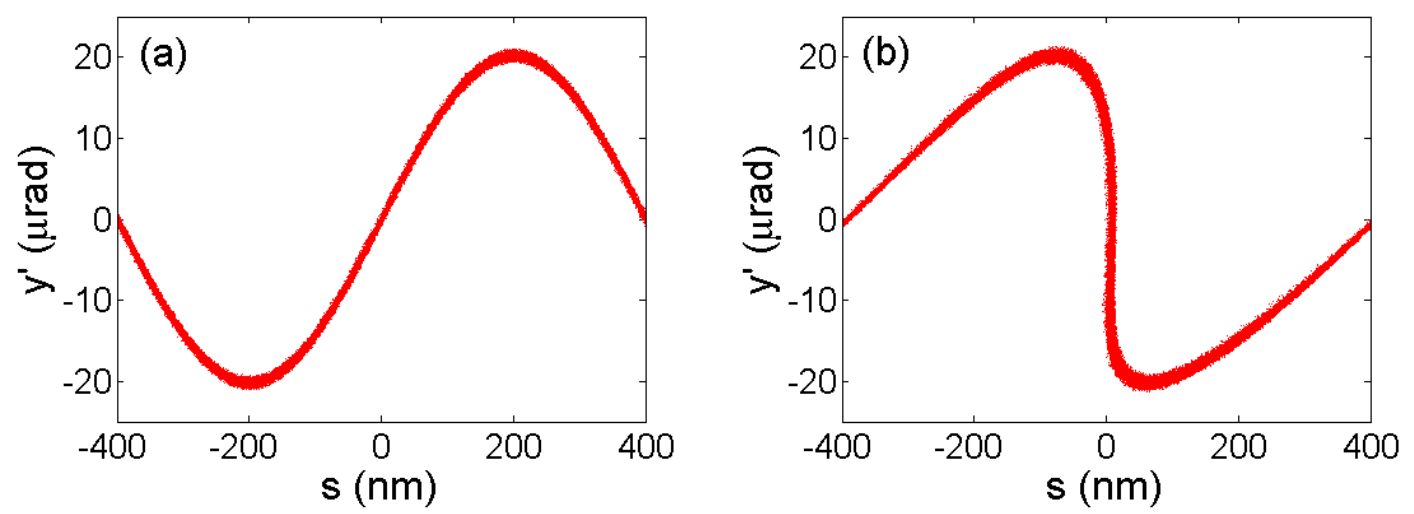

FIG. 2: Phase space evolution in the angular-modulated scheme:(a) $y^{\prime}$ vs $s$ at the exit from the modulator after interaction with the laser; (b) $y^{\prime}$ vs $s$ at the entrance to the radiator after passing through the chicane.

longitudinal coordinate $s$. Using the beam distribution at the exit of the chicane, the bunching factor for various harmonic numbers are calculated and shown in Fig. 3. The agreement between the simulation and the 1-D analytical formula is excellent for small harmonic number, but the difference becomes prominent as $n$ exceeds 20 . The deviation is mainly due to the second order transportation effects. Since strong quadrupoles are used to achieve a vanishing $R_{56}$, the path length differences for the particles with different betatron amplitudes may have considerable values which may cause degradations to the density modulation. Tracking a bunch with zero initial length through the whole beam line (laser-beam interaction is neglected and the bending angles set to zero) yields a rms bunch length of about $1.5 \mathrm{~nm}$ at the exit of the chicane. The smearing effect is negligible for the small harmonic number, but it tends to reduce the bunching factor for large harmonic number of which the wavelength is on the order of nm.

The bunching factor for the 50th harmonic is about $11 \%$ and that for the 80th harmonic is about $5 \%$. To generate temporally coherent radiation, one may require the bunching factor to be larger than 1\% [12] in order to effectively suppress the shot noise. This limits the harmonic number to about $n=120$ in our example. It can be anticipated that powerful coherent radiation at the wavelength $\lambda / n$ can be generated when the radiator is tuned to this wavelength. Here we consider a short radiator with a period length of $8 \mathrm{~cm}$ and the number of periods are assumed to be 25. Simulation with the code GENESIS [13] for the PEP-X beam with bunching factor of $11 \%$ yields a peak power of about $3 \mathrm{MW}$ at $16 \mathrm{~nm}$ after 


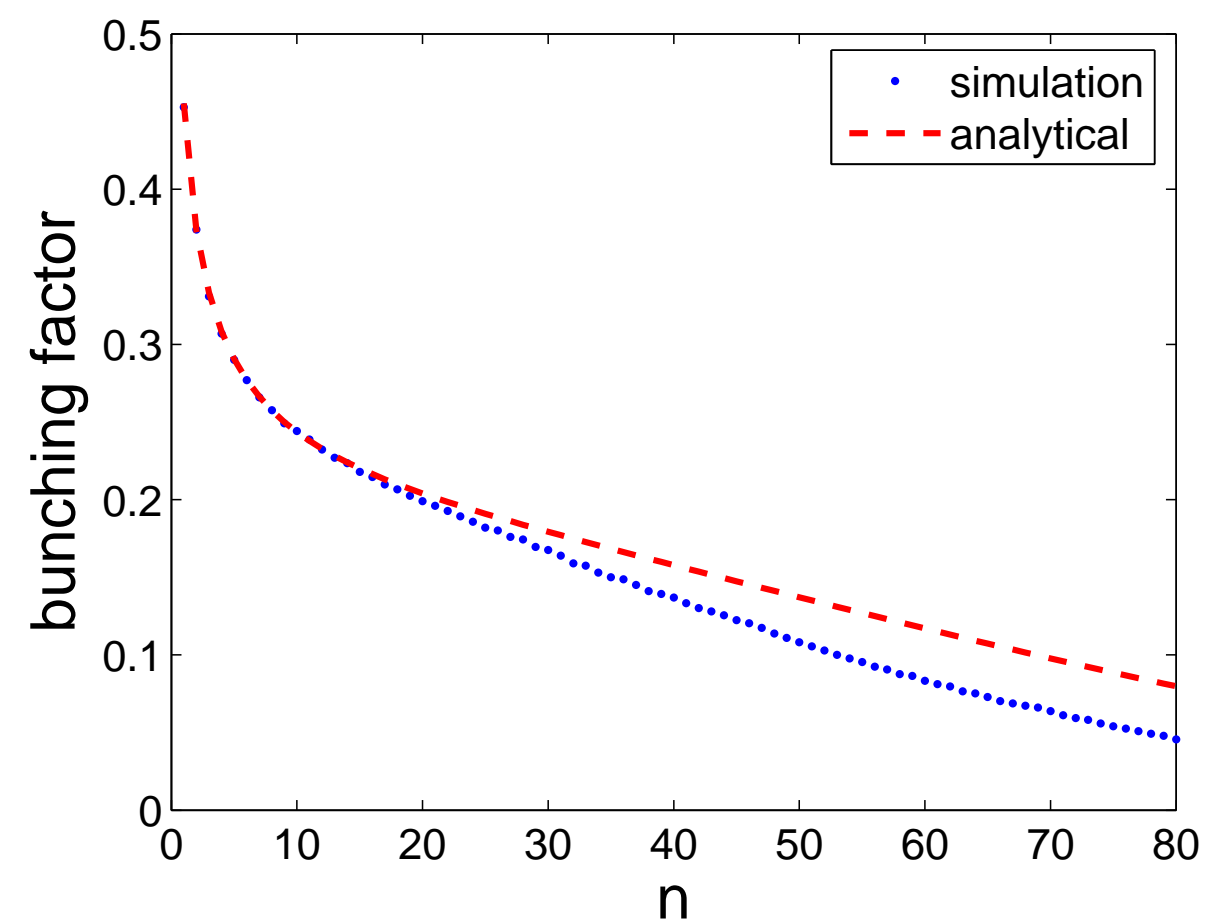

FIG. 3: Bunching factor for various harmonic numbers. The red curve is the result from the 1-D analytical formula Eq. (6) and the blue dots are the results from ELEGANT simulation with second order transportation effects included.

sending the beam through the radiator. The rms pulse duration for the $16 \mathrm{~nm}$ radiation is estimated to be about 35 fs (assuming the seed laser has an rms duration of $100 \mathrm{fs}$ ) and the number of photons contained in the pulse is on the order of $10^{10}$. The coherent radiation should allow one to perform many challenging experiments which require narrow-bandwidth radiation.

It should be pointed out that the proposed scheme relies on a low vertical emittance beam and also a longer beam line is needed to bunch the beam than that is required for the standard scheme where only a small chicane is needed. Depending on the vertical emittance required, the vertical dispersion generated by the chicane may have to be corrected elsewhere in the ring. Nontheless, the proposed scheme is particularly suited for the next generation SR such as the proposed PEP-X at SLAC where both low vertical emittance beam and long straight sections will be available. The proposed technique may be applied to some of the existing SR, too. For instance, straight sections with length of $12 \mathrm{~m}$ are available at SSRF at Shanghai. And typically one can achieve a low vertical emittance by operating the 
SR in a low coupling parameter regime. For instance, it has been demonstrated in ALS at LBNL that by reducing the coupling parameter to $0.1 \%$ a vertical emittance of $5 \mathrm{pm}$ can be achieved [14].

The shortest wavelength achievable with the proposed technique is limited by the intrinsic divergence of the beam (if not limited by the laser power considering the rapid development in laser technology) and the second order transportation effects. The intrinsic divergence of the beam washes out any fine structures shorter than $\sigma_{y}^{\prime} R_{54}$ which is about $1.8 \mathrm{~nm}$ in our example. For a given angular modulation amplitude, one may actually increase the harmonic number by reducing the intrinsic vertical divergence which can be realized by increasing the vertical beta function at the modulator. The second order transportation effects may be mitigated by using a longer chicane with weaker quadrupoles. In principle, the proposed technique should allow one to extend the harmonic number to a few hundred without significantly perturbing the beam dynamics of the SR. Pushing the wavelength to $\mathrm{nm}$ level should significantly enhance the capabilities of SR and eventually lead to more breakthroughs in ultrafast sciences.

We thank A. Chao, Y. Nosochkov, G. Stupakov, M. Woodley and J. Wu for helpful comments and discussions. This work was supported by the US DOE under Contract No. DE-AC02-76SF00515.

[1] B. Girard, et al., Phys. Rev. Lett, 53,2405 (1984).

[2] L.-H. Yu, Phys. Rev. A, 44, 5178 (1991).

[3] L.-H. Yu, et al., Science, 289, 932 (2000).

[4] M. Labat, et al., Eur. Phys. J. D, 44, 187 (2007).

[5] G. De Ninno, et al., Phys. Rev. Lett, 101, 053902 (2008).

[6] A. Zholents and M. Zolotorev, New J. Phys, 10, 025005 (2008).

[7] K. Brown, SLAC-report-075, SLAC, (1982).

[8] Z. Huang, et al., Nucl. Instrum. Methods Phys. Res., Sect. A 593, 120 (2008).

[9] G. Priebe, et al., Laser Part Beams, 26, 649 (2008).

[10] W. Leemans, et al., Nature Phys, 2, 696 (2006).

[11] M. Borland, "Elegant: A flexible SDDS-compliant code for accelerator simulation," Ad- 
vanced Photon Source LS-287, September, (2000).

[12] E. Saldin, et al., Opt. Commun. 202, 169 (2002).

[13] S. Reiche, Nucl. Instrum. Methods Phys. Res., Sect. A 429, 243 (1999).

[14] C. Steier, et al., Proceedings of PAC03, p3213, (2003). 